\title{
Erratum to "Inflammation and Its Role in Prostate Cancer" [Journal of Cancer Therapy 5 (2014) 836-844]
}

\author{
Rakesh K. Mishra, Ashutosh K. Tewari* \\ Department of Urology, Icahn School of Medicine at Mount Sinai, New York, USA \\ Email: "ashtewari@mountsinai.org
}

Received 18 June 2014; revised 13 July 2014; accepted 5 August 2014

Copyright (C) 2015 by authors and Scientific Research Publishing Inc.

This work is licensed under the Creative Commons Attribution International License (CC BY). http://creativecommons.org/licenses/by/4.0/

c) (i) Open Access

The original online version of this article (R. K. Mishra and A. K. Tewari (2014) Inflammation and Its Role in Prostate Cancer, 2014, 5, 836-844. http://dx.doi.org/10.4236/jct.2014.59092) was published in August, 2014. The author wishes to correct the following error due to uploading of unedited draft resulting in typos (including title) and missing sections and Figure 3.

\begin{abstract}
Inflammation is associated with various cancers, and plays a significant role in prostate cancer genesis including initiation, promotion, malignant conversion, invasion, and metastasis. While several studies posit an important role of inflammation in prostate growth, the exact mechanisms are not known. The extant basic and clinical research in the area, trying to unravel the etiology of prostatic inflammation and its signaling pathway, may help to develop novel therapeutic interventions against prostate cancer development triggered by inflammation.
\end{abstract}

\section{Introduction}

Inflammation, a physiological process in response to infection, chemical toxicity, ischemia or autoimmune injury, induces cellular and genomic damage, as well as promotes cellular turnover. Prolonged inflammatory microenvironment supplies chronic exposure to biological modifiers such as reactive nitrogen and oxygen species, reactive aldehydes, cytokines, chemokines, and growth factors. Over time these substances could alter normal cellular homeostasis and lead to uncontrolled proliferative response and genomic instability; it also increases the risk of prostate cancer development [1]. Prostate cancer (PCa) is the most commonly diagnosed cancer in men (27.6\% of all new cases), and is responsible for the second highest number of cancer-related deaths in men (10.7\% 
of all cancers) in the United States [2] [3].

While epidemiologic link between inflammation and cancer was suspected in 1863 by Rudolf Virchow, who described "lymphoreticular infiltrate" in the cancer area. Recent estimates suggest that about $20 \%$ of all human cancers are linked to inflammation [4]. Prominent examples are strong cancer associations with chronic gastritis, hepatitis, prostatitis [5].

There is an expanding body of literature suggesting a link between inflammation and prostate cancer [6]. The prevalence of both prostate cancer and prostatic inflammation is not only epidemic but also run in parallel in the United States. Prostatic carcinogenesis is multi-factorial in which genetic underpinnings interact with environmental exposures of which many of them are shared between inflammation and PCa [7]-[9].

Inflammation involves both an innate and adaptive immune response by producing a large number of cytokines, reactive oxygen (ROS), and reactive nitrogen species (RNS) [10]. This process eliminates pathogens and repairs tissue damage, and activates the adaptive immune response. Thus, inflammation could be seen a double-edged sword that mostly serves as a host defense and, usually, is a self-limiting process; however, inflammation could also become damaging and carcinogenic if not resolved properly. Chronic inflammation is linked to tumour progression by several mechanisms, including increased cell proliferation, angiogenesis, and evasion from apoptosis [10].

\section{Inflammation and Prostate Cancer}

Coordinated chemical signals are induced following injury that promotes "healing" of damaged tissues [12] [13]. Early responses include vascular permeability and activation and migration of leukocytes towards the injury site. This is mediated by a gradient of secreted chemokines towards the site of inflammation [14]. Normally, inflammation is a self-limiting process that is controlled by production of anti-inflammatory cytokines. The cytokine/chemokine balance at the inflammatory site is important in the development of chronic disease. Deregulation of which can lead to prolonged inflammation with chronic exposure to cytotoxic mediators [15]. Chronic inflammation can be caused by a variety of factors, including microbial infections, chemical irritants, and foreign particles. The chronicity of inflammation increases the risk of associated carcinogenesis [16].

The molecular primers of pathogenesis of cancer related inflammation are complex and involve interplay between tumor and its microenvironment. To address the details of transition from inflammation to cancers and the further development of inflammation associated cancers, it is necessary to investigate specific roles of key regulatory process involved in this process. Prolonged activation of NF- $\kappa \mathrm{B}$ has been implicated in several cancers including PCa through the induction of pro-inflammatory cytokines, such as IL-6, TNF- $\alpha$, and COX-2, and this may also exacerbate genomic instability by promoting release of ROS and RNS.

1) The oxidative stress imbalance in the prostrate tumor, and

2) The cytokine \& chemokine orchestration in prostate cancer.

\subsection{The Oxidative Stress Imbalance in the Prostrate Tumor}

The oxidative stress associated with infection and inflammation can cause prostate carcinogenesis through the induction of iNOS (inducible nitric oxide synthase), leading to reactive nitrogens and oxygen reactive species activation.

At the site of inflammation phagocytic cells (e.g. neutrophils and macrophages) generate reactive oxygen and nitrogen species. These cells also synthesize and secrete large quantities of growth factors and potent angiogenic factors, cytokines, and proteases. These are important mediators in the tissue regeneration process, but can also potentiate neoplasia. Prostaglandins, cytokines, nuclear factor NF- $\kappa \mathrm{B}$, chemokines and angiogenic factors are the main molecular mediators and free radical species derived from oxygen (ROS) and nitrogen (RNS) are the main chemical effectors [17] [18].

Human cells have the following coping mechanisms: direct antioxidant enzymes, proteases, phospholipases, and lipid and water-soluble antioxidants [19] [20]. Inflammation can cause proliferation within prostatic tissue through compensatory cellular proliferation with resulting hypertrophy. Prostatic inflammation generates free radicals (ROS, NOS) through macrophages and neutrophils which can induce hyperplastic transformations [21] [22].

The macrophages in the tumor microenvironment produce reactive radicals causing DNA damage and genetic alterations such as; p53 and AR mutations, hypermethylation of the CpG island of gluthathione S tranferase-P1 (GSTP1) promoter, decreased activity of manganese superoxide dismutase (MnSOD) and increased expression of NADPH oxidase 1 [23]. 
ROS are endogenously generated during cellular metabolic processes but can also come from external sources. Excessive ROS production or impairment of antioxidant defense systems can induce oxidative stress and promote development of various cancers, including prostate cancer, because oxidative stress regulates cellular fate in various systems. ROS are considered to be tumor initiators/promoters given the potential for induction of DNA damage. Furthermore, signaling pathways in response to intracellular changes in ROS levels may trigger proliferation, apoptosis and senescence, events highly implicated in all the stages of the carcinogenic process [24] (Figure 1).

The NF- $\kappa$ B family of transcription factors is critical in carcinogenesis [25] through modulation of genes involved in cell survival, proliferation, angiogenesis, and invasion [26]. NF- $\kappa \mathrm{B}$ is a dimer formed by proteins of the Rel family (RelA/p65, RelB, c-Rel, NF- $\kappa \mathrm{B} 1 / \mathrm{p} 50$, and NF- $\kappa \mathrm{B} 2 / \mathrm{p} 52$ ) that is retained in the cytoplasm as a complex with inhibitory $\mathrm{I} \kappa \mathrm{B}$ proteins that is subsequently trans-located into the nucleus where it binds specific elements in the promoters of NF- $\kappa$ B-responsive genes [27]. Hyperactivation of NF- $\kappa \mathrm{B}$ is a hallmark of chronic inflammation and has been widely linked to cancer. As mentioned before inflammation contributes to prostate cancer development via multiple mechanisms such as oxidative stress, genomic instability, and DNA damage. Indirectly it increases levels of proinflammatory factors such as tumor necrosis factor $\alpha$ (TNF $\alpha)$ [27]. NF- $\kappa \mathrm{B}$ hyperactivation results from enhanced production of tumorpromoting cytokines, stimulation of growth factor receptors, and aberrant activation of upstream NF- $\kappa$ B kinases such as IKK and NF- $\kappa$ B-inducing kinase (NIK).

Several lines of evidence suggest NF- $\kappa$ B pathway is dysregulated in prostate cancer and has been implicated in the progression to the lethal androgen-independent state. Constitutive NF- $\kappa \mathrm{B}$ activation has been reported in several prostate tumors and since nuclear NF- $\kappa \mathrm{B}$ is predominantly observed in organ-confined tumors, it suggests it to be an early event in prostate cancer development [28] [29]. NF- $\kappa$ B mediates the effect of proinflammatory cytokines such as TNF $\alpha$, IFN $\gamma$, and IL-1 $\beta$ [30]. Binding of TNF $\alpha$ to its receptor, leads to the recruitment of adaptor proteins, that ultimately regulates NF- $\kappa \mathrm{B}$ activation and transcriptional activation of inflammatory, and anti-apoptotic genes.

Overall, available evidence confirms that NF- $\kappa \mathrm{B}$ activation is a key event in PCa pathogenesis and aggressiveness through EMT induction (see next section). Constitutive or induced activation of NF- $\kappa \mathrm{B}$ is critical in providing a positive feedback signal to immune cells in the tumour microenvironment. This increases the production of molecular mediators such as various proinflammatory cytokines \& chemokines, which contribute to carcinogenic and inflammatory processes (Figure 2).

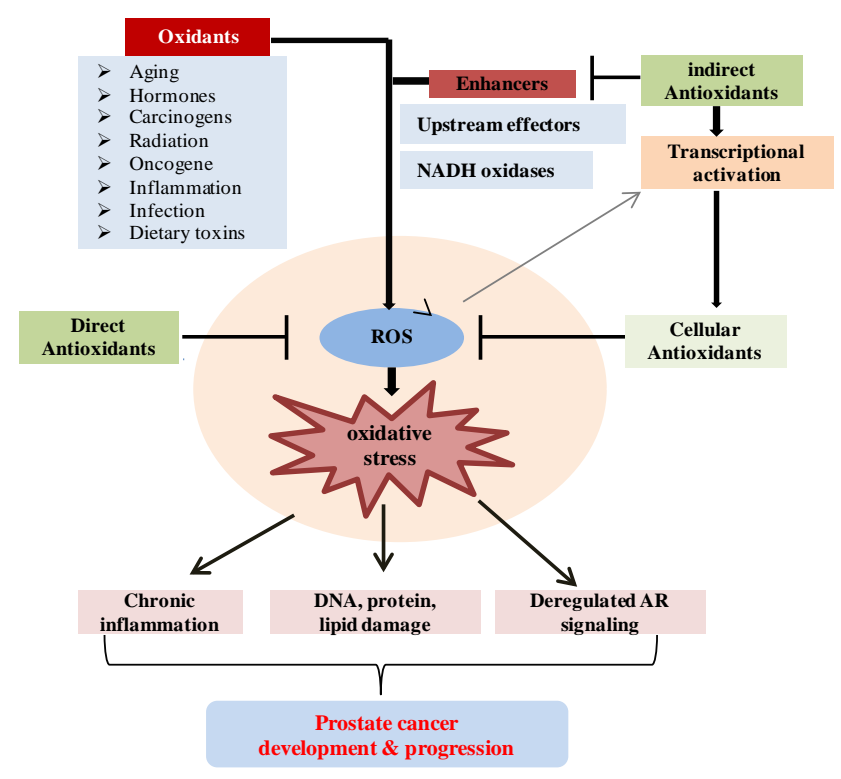

Figure 1. Inflammation and PCa. Inflammation can be triggered by a variety of stresses and stimuli such as infection, radiation, inflammatory stimuli and changes in hormonal status. Cancer-related inflammation is characterized by the presence of inflammatory cells at the tumor sites and the over expression of inflammatory mediators such as cytokines, chemokines, reactive oxygen species (ROS), and nitric oxide (NO) in tumor tissue. These pro-inflammatory mediators promote malignant transformation and prostate cancer progression. 


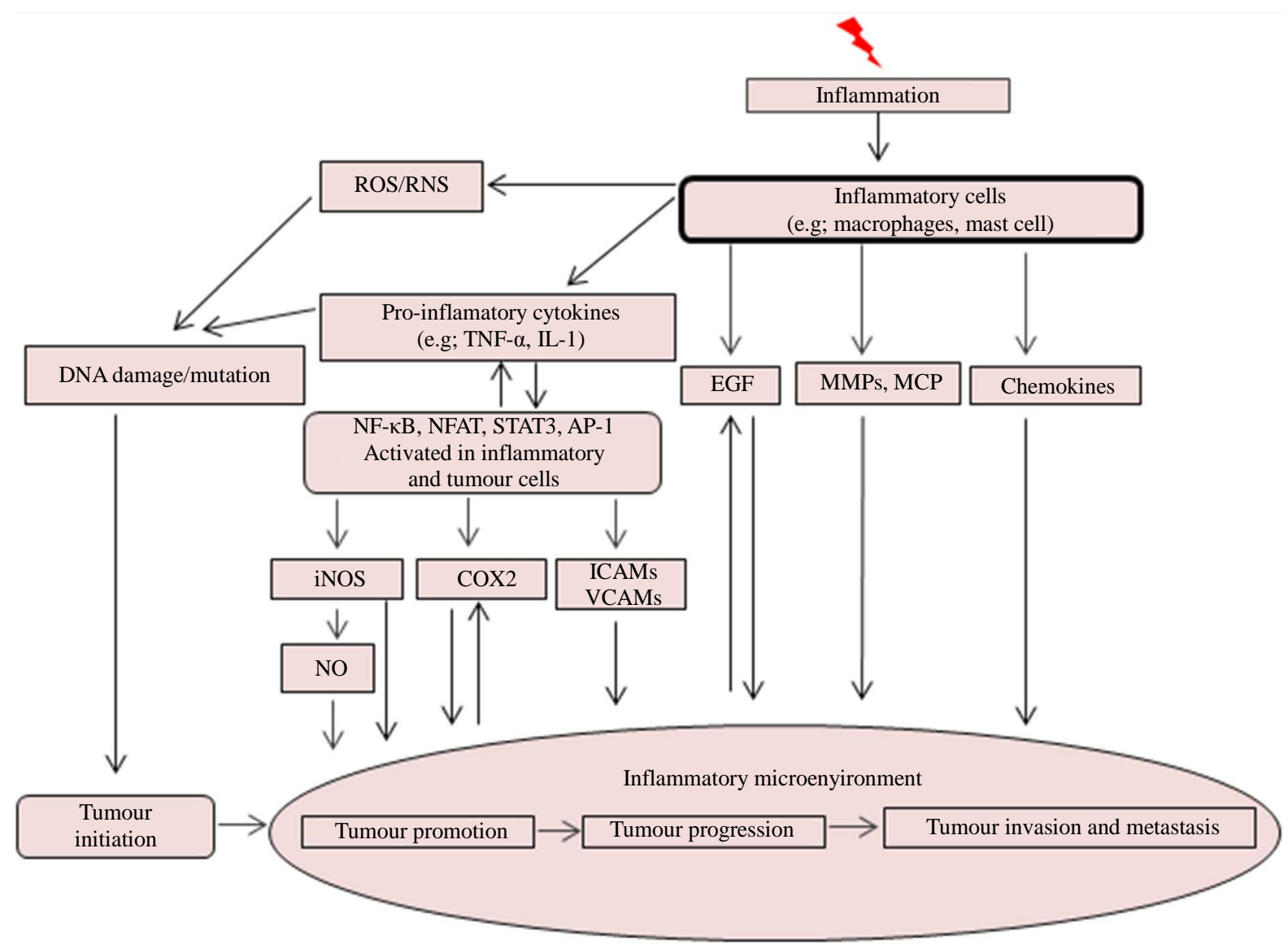

Figure 2. Summary of mechanisms for the involvement of inflammation in cancer development.

\subsection{The Cytokine Orchestration in Prostate Cancer}

Cytokines are a family of cell-signaling molecules (proteins, peptides, or glycoproteins) that are secreted by various cell types in the tumor microenvironment, are used extensively in intercellular communication and can impact prostate cancer growth [31].

Cytokines, including TNF- $\alpha$, IL, growth factors, and differentiation factors (colonystimulating factors), contribute to the progression of prostate tumors of altered cells at the inflammatory site [32].

Several evidences suggest that TNF and chemokines are mediators of inflammation and prostate cancer [33]. TNF, product of activated macrophages and tumor cells, binds to membrane-bound receptors and plays a critical role in both tissue destruction and damage recovery. Thus, it helps in maintaining the reversibility of microenvironments, stimulating cellular change, and tissue remodeling [33].

In relapsing cancers TNF- $\alpha$ is not released from prostate cancer cells rather from associated macrophages and in hormone-refractory cases serum TNF- $\alpha$ levels are higher than untreated patients [34] [35]. While the relationship between TNF- $\alpha$ and hormone responsiveness has not been explored, androgen-insensitive prostate cancer cells have proven to be TNF- $\alpha$ insensitive [36]. The recent publication from Davis et al. [37] explains the dichotomy of TNF- $\alpha$ effect on the control of apoptosis in prostate cancer cells through proposed physiologic role for TNF- $\alpha$ in prostate regression after androgen withdrawal.

Inflammatory cytokines have also been reported to facilitate the spectrum of prostate tumor development particularly IL-6, which is a multifunctional cytokine, produced by not only by inflammatory cells, but also osteoblasts and even prostate cancer cells. Several lines of clinical and experimental evidence suggest that IL-6 contributes to prostate cancer progression. Both, early and advanced prostate cancer display high expression levels of IL-6 [38]. There is accumulating evidence linking IL-6 levels to prostate cancer stage as IL-6 serum levels were found to be strongly elevated and positively correlated to tumor load [39] [40]. In other study Shariat et al. 
showed that LNCaP cells continuously exposed to IL-6 develop increased invasiveness as assessed by chamber-based migration assay develop neuroendocrine features when exposed to IL-6 [41] [42].

It is important to note IL-6 modulates the growth of hormone-refractory PCa cells, but not of hormone-dependent cell lines [43].

IL-6 has also been implicated in other aspects of prostate cancer pathophysiology such as tumorigenesis in the prostate microenvironment through the activation of Janus kinase (JAK) and signal transducers and activators of transcription (STAT), signaling [44]. IL-6 also stimulates autocrine activation of insulin-like type I growth factor receptor (IGF-IR) to confer tumorigenesis. Furthermore IL-6 can also signal through MAPK and phosphatidylinositol-3 kinase (PI3K) pathways [44]-[48].

Cytokines like IL-8 and IL-17 are also involved in prostate cancer as, IL-8, an inflammatory chemokine, promotes progression of human solid tumors by its ability to regulate the expression of matrix metalloproteinases (MMPs) [49]. Numerous studies have demonstrated a correlation between MMPs, IL-8, and CPa as increased levels were associated with high Gleason scores and metastatic disease. High levels of IL-8 lead to an increase in MMP-9 expression, which directly increases the tumor grade and metastasis [49] [50].

Steiner et al. have shown that $58 \%$ of human malignant prostate tissues have an increased level of IL-17 mRNA implying that IL-17 acts directly on the prostate tumor cells and promotes tumorigenesis.

Taken together, experimental studies have validated the crucial role of various pro/anti-inflammatory cytokines in prostate cancer progression and development.

\section{Inflammation and EMT}

While analyzing role of various molecular mechanisms implicating role of inflammation in prostate carcinogenesis we realize that epithelial to mesenchymal transformation (EMT) also plays an important role in helping cancer become more invasive and metastatic. EMT is an embryonic phenomenon where there is a need for cells to move and invade surrounding tissues. This EMT phenomenon has been observed in many aggressive cancers. Accumulating evidences suggest that EMT could be induced by inflammatory process involving tumor micro-environment and actually help cancer become more aggressive and metastatic. Various proposed pathways for such Inflammation-EMT induction are summarized in Figure 3. In future we may be able to target EMT and possibly limit invasive morphology of prostate cancer.

\section{Conclusion and Future Direction}

We have summarized the existing data on inflammation and prostate cancer but must admit the story is still evolving and many new associations are waiting to be discovered. There appears to be an intriguing feedback loop between cytokines and NF- $\kappa \mathrm{B}$ that has a role in prostate cancer. Future answers will come by study from animal models for inflammation-derived cancers and a combination of molecular approaches. We believe that better clarification of the mechanisms linking inflammation and prostate cancer will yield discoveries in preventive and therapy.
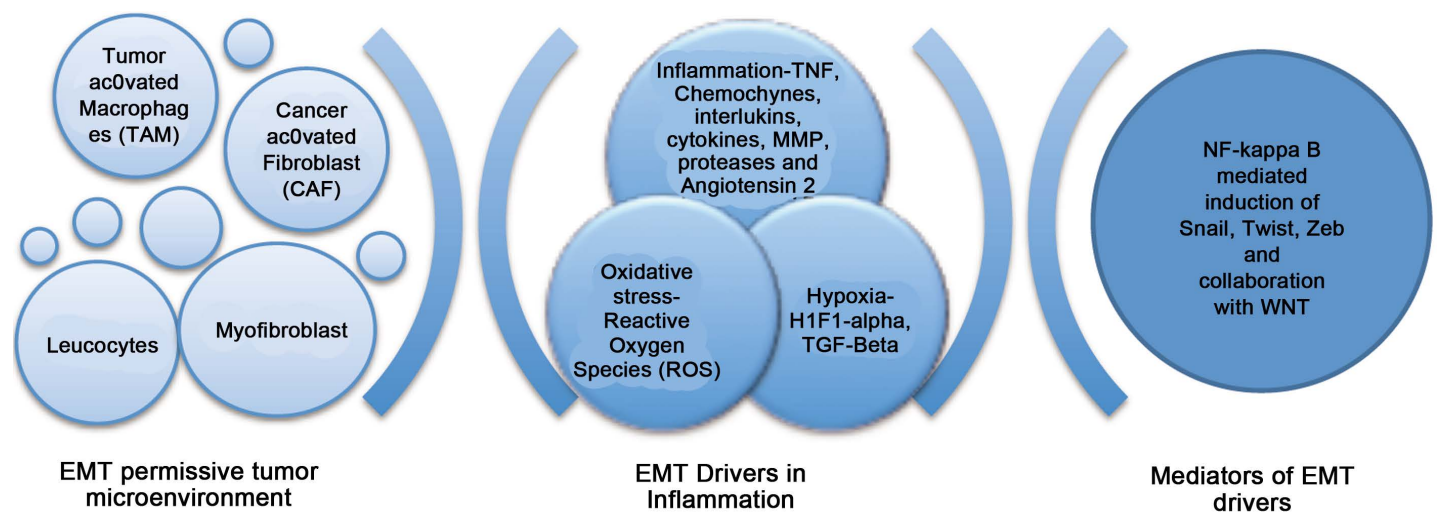

Figure 3. Simplistic pathways for Inflammation induce EMT. Inflammation promoted by lymphocytes or fibroblasts within the tumor micro-environment, though various effectors, activates the mediators or drivers of EMT. 